\title{
Atomic-scale Structural Imaging of Interfacial Defects in GaAs(001)-based Heterostructures
}

Abhinandan Gangopadhyay ${ }^{1}$, Thomas J. Rotter ${ }^{2}$, Ganesh Balakrishnan ${ }^{3}$ and David J Smith ${ }^{4}$

${ }^{1}$ Arizona State University, United States, ${ }^{2}$ University of New Mexico, United States, ${ }^{3}$ The University of New Mexico, United States, ${ }^{4}$ Arizona State University, 85281, Arizona, United States

Interfacial defects in lattice-mismatched semiconductor heterostructures are commonly formed to plastically relax strained epitaxial layers. The atomic configurations at the cores of these defects determine the electronic properties and thus indirectly impact device performance. The sub-Ångstrom resolution offered by the recent generation of aberration-corrected high-resolution electron microscopes facilitates atomic-scale characterization of defects. In this work, interfacial defects in GaAs(001)-based heterostructures, especially in high-mismatched $\mathrm{GaSb} / \mathrm{GaAs}(001)$ heterostructures, are studied using a probe-corrected scanning transmission electron microscopes (STEM). The semiconductor heterostructures were grown by molecular beam epitaxy. The TEM samples were prepared using mechanical polishing, dimpling, and argon ion-milling at liquid nitrogen temperature. STEM images were recorded using a JEOL ARM-200F microscope operated at $200 \mathrm{kV}$. The probe convergence angle was 22 $\mathrm{mrad}$, and the inner and outer angles for high-angle annular dark-field (HAADF)-STEM imaging were 90 and $150 \mathrm{mrad}$, respectively.

Strain relaxation in the high-mismatched $\mathrm{GaSb} / \mathrm{GaAs}$ heterostructure was primarily carried out by Lomer edge dislocations. Three different types of Lomer dislocations were observed, namely shuffle-set Lomer dislocations consisting of five- and seven-membered atomic rings (shown in Figure 1), leftdisplaced glide-set Lomer dislocations consisting of six- and eight-membered atomic rings (shown in Figure 2(a)) and right-displaced glide-set Lomer dislocation consisting of six- and eight-membered atomic rings with an atomic step (shown in Figure 2(b)). The average separation between the Lomer dislocations at the interface was measured to be $6.0 \pm 0.5 \mathrm{~nm}$. Only a relative few of the Lomer dislocations were associated with monolayer steps indicating that interfacial steps were not a prerequisite for the dislocation formation. In addition to Lomer dislocations, both perfect and dissociated $60^{\circ}$ dislocations were also observed. The presence of single atomic columns at the core of the perfect $60^{\circ}$ dislocation and at the core of the $30^{\circ}$ Shockley partial dislocation associated with the dissociated $60^{\circ}$ dislocation indicated that both of these dislocations belonged to the glide set. Among these different types of dislocations, the shuffle-set Lomer dislocation was the most prevalent. Detailed Burgers circuit analysis and contrast analysis of atomic-resolution STEM images of these interfacial dislocations will be described.

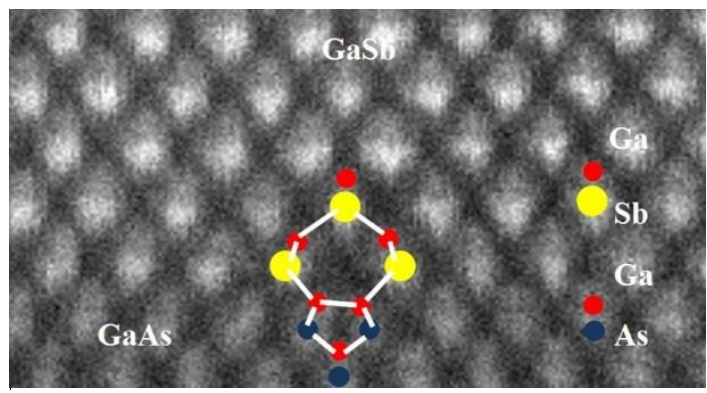

Figure 1. Aberration-corrected HAADF-STEM image of shuffle-set Lomer dislocation with proposed atomic arrangement overlaid on the image. 

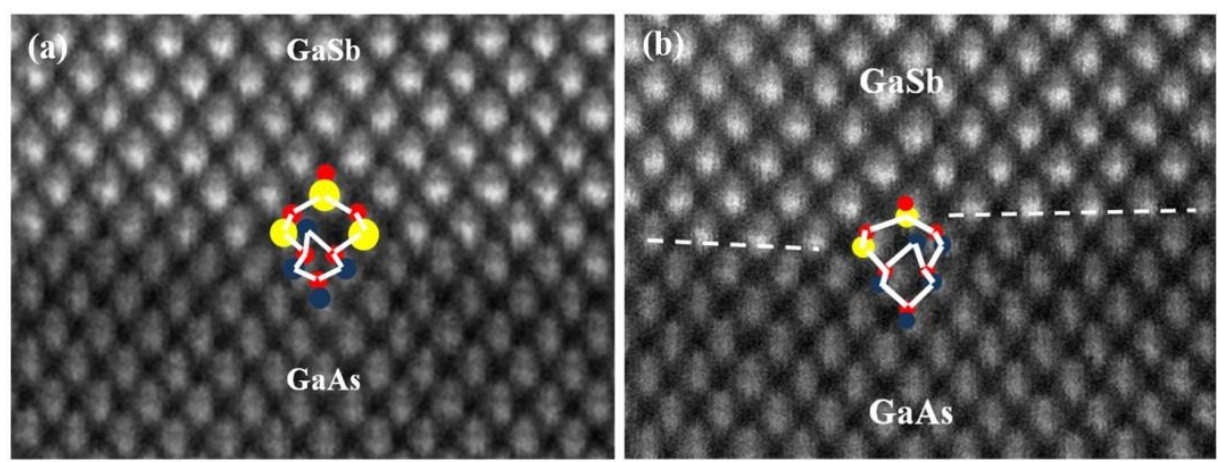

Figure 2. Aberration-corrected HAADF-STEM images: (a) left-displaced glide-set Lomer dislocation with proposed atomic arrangement overlaid on the image; and (b) right-displaced glide-set Lomer dislocation with proposed atomic arrangement overlaid on the image. The white dotted line shows the stepped GaSb/GaAs interface.

\section{References}

This work was supported by the National Science Foundation and the Department of Energy under NSF CA No. EEC-1041895. The authors acknowledge the use of facilities within the John M. Cowley Center for High Resolution Electron Microscopy at Arizona State University. 